\title{
Relationship of serum ATPase activity and the levels of neuron- specific enolase, S100B and B-cell lymphoma/leukemia-2 with cognitive function after epileptic seizure
}

\author{
Yi Zhang ${ }^{1}$, Shanshan Zhang ${ }^{1}$, Yifei Ji ${ }^{1}$, Xu Yang ${ }^{1}$, Pingping Liu ${ }^{1}$, Gang Yu ${ }^{2}$ \\ ${ }^{1}$ Department of Neurology, Nanchong Central Hospital, Nanchong, China; ${ }^{2}$ Department of Neurology, The First Affiliated Hospital of Chongqing \\ Medical University, Chongqing, China \\ Contributions: (I) Conception and design: Y Zhang, G Yu; (II) Administrative support: S Zhang, Y Ji; (III) Provision of study materials or patients: Y \\ Zhang, S Zhang, Y Ji, X Yang, P Liu; (IV) Collection and assembly of data: All authors; (V) Data analysis and interpretation: Y Zhang, Y Ji, X Yang, \\ P Liu, G Yu; (VI) Manuscript writing: All authors; (VII) Final approval of manuscript: All authors. \\ Correspondence to: Gang Yu. The First Affiliated Hospital of Chongqing Medical University, No.1, Youyi Road, Yuanjiagang, Yuzhong District, \\ Chongqing 400000, China. Email: wing_z_y3@126.com.
}

Background: To analyze the relationship of serum ATPase activity and the levels of neuron-specific enolase (NSE), S100B protein and B-cell lymphoma/leukemia (Bcl)-2 with cognitive function in patients after epileptic seizure.

Methods: A total of 65 epilepsy patients were enrolled as the epilepsy group from the hospital between May 2017 and February 2019. A control group was formed by 30 healthy volunteers at the same period and in the same area whose basic data matched with those of the epileptic patients. Cognitive function was evaluated using the Montreal Cognitive Assessment Form (MoCA). Time and frequency of epileptic seizures were recorded in epileptic group. Within 6 hours following the attack, blood tests were carried out to measure the hydrolysis rates of serum adenosine triphosphate (ATP), adenosine diphosphate (ADP), adenosine monophosphate (AMP), phosphodiesterase (PDE) activity, and levels of serum NSE, S100B and Bcl-2. The relationship of these indexes with the time of epileptic seizure and MoCA total score was analyzed.

Results: The epilepsy group included 18 cases (27.69\%) with cognitive dysfunction. The time and frequency of epileptic seizure in the patients with cognitive dysfunction was significantly higher than that of patients without cognitive dysfunction, while the total MoCA score was significantly lower with cognitive dysfunction than that without $(\mathrm{P}<0.05)$. The total MoCA score of epileptic patients with and without cognitive dysfunction was significantly lower than that of the control group $(\mathrm{P}<0.05)$. The hydrolysis rates of ATP, ADP and AMP, PDE activity, and serum NES, S100B and Bcl-2 levels in patients with cognitive dysfunction were significantly higher than those without cognitive dysfunction $(\mathrm{P}<0.05)$. The above serum indexes of the patients were significantly higher than those of people in the control group $(\mathrm{P}<0.05)$. Spearman's correlation analysis showed that hydrolysis rates of serum ATP, ADP and AMP, PDE activity, and serum NES, S100B and Bcl-2 levels were positively correlated with time and frequency of epileptic seizure $(\mathrm{P}<0.05)$, and negatively correlated with total MoCA score $(\mathrm{P}<0.05)$.

Conclusions: The cognitive function of epilepsy patients is significantly decreased compared with that of healthy individuals. Serum ATPase activity, along with serum NES, S100B and Bcl-2 levels, is associated with time and frequency of epileptic seizure and cognitive function after attack.

Keywords: Epilepsy; ATPase activity; neuron-specific enolase (NSE); B-cell lymphoma/leukemia (Bcl); cognitive function

Submitted Jul 08, 2020. Accepted for publication Aug 28, 2020.

doi: 10.21037/apm-20-1494

View this article at: http://dx.doi.org/10.21037/apm-20-1494 


\section{Introduction}

Epilepsy, a chronic onset neurological dysfunction disease, is mainly caused by sudden, disordered, and excessive neuronal discharge, which is characterized by spontaneous and periodic disruption of normal electrical activity in the brain and results in recurrent seizures (1). Globally, approximately 50 million people are diagnosed with epilepsy annually, with China accounting for about $18 \%$ of these patients (2). After stroke, epilepsy has become the second most common central nervous system disease in China. Studies (3) have pointed out that about $30-40 \%$ of patients with epilepsy experience varying degrees of cognitive dysfunction, such as inattention, decreased execution and intelligence, disorders of language and memory. The human adenosine system has been clinically confirmed to be involved in the processes of synaptic transmission and neuronal excitation. The well-known adenosine members, including adenosine triphosphate (ATP), adenosine diphosphate (ADP), and adenosine monophosphate (AMP), have neuroprotective and anticonvulsant effects; as a result, this system is known as the "endogenous antiepileptic system", and it has become a new hotspot in epilepsy research (4). At the same time, with the continuously expanding in-depth clinical research on epilepsy, ATPase activity and the levels of neuron-specific enolase (NSE), S100B protein and B-cell lymphoma/leukemia-2 (Bcl-2) have been found to be associated with seizures and development of epilepsy (5). However, systematic studies on cognitive status after epileptic seizures are still rare, and the relationship between these indicators and cognitive function in patients with epilepsy has yet to be clarified. Therefore, we aim to perform a novel study to analyze the relationships of serum ATPase activity and the levels of NES, S100B and Bcl-2 with cognitive function in epileptic patients, with a view to providing a basis for the clinical diagnosis, evaluation, and prevention of the disease, as well as for related research. We present the following article in accordance with the MDAR reporting checklist (available at http://dx.doi.org/10.21037/ apm-20-1494).

\section{Methods}

\section{The study objects}

A total of 65 patients with epilepsy who were diagnosed in our outpatient and inpatient departments between May 2017 and February 2019 were enrolled as the epilepsy group. The group included 37 males and 28 females with a mean age of $41.88 \pm 8.82$ years (range, $25-75$ years). The mean years of schooling for the group was 6-16 years, with an average of $(12.12 \pm 2.17)$ years. And the course of disease was 2 to 12 years, with an average course of $(6.33 \pm 1.50)$ weeks. The key criteria for inclusion in the study were as follows: (I) newly diagnosed epilepsy with 2 or more generalized seizures (including convulsions, tonic-clonic seizure, myoclonus, atony, and loss of consciousness); (II) combined with clinical manifestations, the diagnosis was confirmed by CT or MRI examination and EEG results, in line with the 1989 International League Against Epilepsy (ILAE) classification and diagnosis of epilepsy syndrome (6); (III) a high degree of family support and patient cooperation; and (IV) well-documented clinical data. The exclusion criteria were as follows: (I) clear hereditary epilepsy; (II) craniocerebral tumors, cerebral infarction lesions, purulent meningitis, viral encephalitis, congenital white matter dysplasia, vascular malformations, or other progressive lesions confirmed by cranial imaging; (III) other mental illnesses, such as autism, attention deficit hyperactivity disorder (ADHD), anxiety, or depression; (IV) genetic metabolic diseases, or Alzheimer's dementia or other diseases that may affect cognitive function; (V) a history of alcoholism or a long-term history of drug abuse; or (VI) Pregnant women.

In addition, 30 healthy volunteers who matched with the basic data of the epilepsy group was enrolled as the control group. The control group included 17 males and 13 females with a mean age of $42.57 \pm 9.31$ years (range, $25-75$ years).

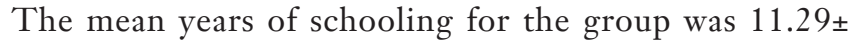
3.49 years (range, 6-15 years). There were no significant differences in gender, age, or years of schooling between the two groups $(\mathrm{P}>0.05)$. Written informed consent was obtained from each study participant before the initiation of the study procedures, and the study protocol and informed consent documents were reviewed and approved by the hospital Ethics Committees. All procedures performed in this study involving human participants were in accordance with the Declaration of Helsinki (as revised in 2013).

\section{Cognitive function assessment}

Montreal Cognitive Assessment (MoCA) (7), which involves visual space and executive function, naming, memory, attention, language, abstraction, delayed recall, and orientation, was used as an assessment tool. The total MoCA score is 30 points, $\geq 26$ points is normal and $<26$ points is judged as cognitive dysfunction. 
Table 1 Comparison of serum ATPase activity between the two groups $[\bar{x}+\mathrm{s}, \mathrm{nmol} /(\mathrm{min} \cdot \mathrm{mL})]$

\begin{tabular}{|c|c|c|c|c|c|}
\hline Group & Sample size & Hydrolysis rate of ATP & Hydrolysis rate of ADP & Hydrolysis rate of AMP & PDE activity \\
\hline \multicolumn{6}{|l|}{ Epilepsy } \\
\hline Without cognitive impairment & 65 & $1.53 \pm 0.21^{\Delta}$ & $0.63 \pm 0.11^{\Delta}$ & $0.72 \pm 0.17^{\Delta}$ & $0.43 \pm 0.08^{\triangle}$ \\
\hline With cognitive impairment & 15 & $2.12 \pm 0.30^{\Delta \Delta}$ & $0.87 \pm 0.18^{\Delta 凶}$ & $0.83 \pm 0.18^{\triangle \Lambda}$ & $0.67 \pm 0.11^{\triangle \Delta}$ \\
\hline$P$ value & & 0.000 & 0.000 & 0.002 & 0.000 \\
\hline
\end{tabular}

Compared with the control group, ${ }^{\Delta} \mathrm{P}<0.05$; compared with the group without cognitive impairment, ${ }^{\Delta} \mathrm{P}<0.05$. ATP, adenosine triphosphate; ADP, adenosine diphosphate; AMP, adenosine monophosphate; PDE, phosphodiesterase.

\section{Determination of serum indicators}

The seizure times of the patients in the epilepsy group were recorded. Fasting venous blood samples $(3 \mathrm{~mL})$ were collected within the 6 hours following the seizure. The same amount of fasting venous blood was collected from the control group subjects in the early morning resting state. All blood samples were centrifuged at 3,000 r/min (centrifugation radius $15 \mathrm{~cm}$ ) for $10 \mathrm{~min}$. The upper serum was collected and stored at $-80{ }^{\circ} \mathrm{C}$ for analysis. (I) ATPase activity: according to the reported method (8), $5 \mu \mathrm{L}$ of serum was added to a total volume of $175 \mu \mathrm{L}$ solution with with $5 \mathrm{mmol} / \mathrm{L}$ of $\mathrm{CaCl}_{2}$ and $40 \mathrm{mmol} / \mathrm{L}$ of Tris-HCl. After preincubation at $37^{\circ} \mathrm{C}$ for $10 \mathrm{~min}, 10 \mu \mathrm{L}$ of ATP, ADP, and AMP was added to determine the hydrolysis, respectively. The hydrolysis $\mathrm{pH}$ values of the ATP, ADP, and AMP were 8.0, 7.2, and 8.9, respectively. ATP, ADP, and AMP hydrolase activity was expressed as the amount of inorganic phosphate (nmol) released per milliliter of serum per minute. Phosphodiesterase (PDE) activity was expressed as the amount of phosphate-nitrophenol (nmol) released per milliliter of serum per minute. (II) NES, S100B and Bcl-2: the serum NES, S100B and Bcl-2 levels were measured by enzyme-linked immunosorbent assay (ELISA). The kit was purchased from Tianjin Jiuding Medical Bioengineering Co., Ltd. (Tianjin, China). The operation steps were performed strictly following the manufacturer's instructions.

\section{Statistical analysis}

The data were processed with SPSS 20.0 statistical software (IBM, USA). Measurement data were presented as $\bar{x} \pm$ SD. Serum ATPase activity and levels of NES, S100B and Bcl-2, as well as the total MoCA score of the two groups were analyzed by independent $t$-test or analysis of variance. The relationship of serum ATPase activity and the levels of NES, S100B and Bcl-2 with seizure time, frequency and total MoCA score was assessed by Spearman's correlation analysis. $\mathrm{P}<0.05$ was considered as statistically significant.

\section{Results}

\section{Results of seizure time and cognitive function evaluation}

Among the 65 patients in the epilepsy group, 18 had cognitive impairment (27.69\%). The seizure time and frequency of the patients with cognitive impairment were $(8.45 \pm 1.85)$ minutes and $(3.96 \pm 0.71)$ times, respectively, which were significantly longer than that of the patients without cognitive impairment $(6.31 \pm 1.74$ minutes) $(\mathrm{t}=4.244$, $\mathrm{P}<0.05)$. The total MoCA score of the patients with cognitive impairment (24.26 \pm 2.70 points) was significantly lower than that of the patients without cognitive impairment $(27.27 \pm 2.36$ points $)(t=4.421, \mathrm{P}<0.05)$. The total MoCA scores of epileptic patients with and without cognitive impairment were significantly lower than that of the control group $(29.05 \pm 1.87$ points $)(t=7.259,3.635, \mathrm{P}<0.05)$.

\section{Comparison of serum ATPase activity between the two groups}

In the epilepsy group, the ATP, ADP, and AMP hydrolysis rates and PDE activity of patients with cognitive impairment were significantly higher than those without cognitive impairment $(\mathrm{P}<0.05)$. As shown in Table 1, the ATP, ADP, and AMP hydrolysis rates and PDE activity of epileptic patients with and without cognitive impairment were significantly higher than those of the control group $(\mathrm{P}<0.05)$. 
Table 2 Comparison of serum NSE, S100B and Bcl-2 levels between the two groups $(\bar{x}+s)$

\begin{tabular}{lcccc}
\hline Group & Sample size & NSE $(\mu \mathrm{g} / \mathrm{L})$ & S100B $(\mathrm{ng} / \mathrm{mL})$ & $\mathrm{Bcl}-2(\mathrm{ng} / \mathrm{mL})$ \\
\hline Control & 30 & $8.47 \pm 2.18$ & $0.23 \pm 0.06$ & $4.27 \pm 0.92$ \\
Epilepsy & & & & \\
$\quad$ Without cognitive impairment & 65 & $16.49 \pm 2.82^{\Delta}$ & $1.16 \pm 0.25^{\Delta}$ & $6.46 \pm 1.31^{\Delta}$ \\
$\quad$ With cognitive impairment & 15 & $18.96 \pm 3.25^{\Delta \Delta}$ & $1.49 \pm 0.27^{\Delta \Delta}$ & $8.52 \pm 1.64^{\Delta \Delta}$ \\
F value & & 110.714 & 244.600 & 61.118 \\
P value & & 0.000 & 0.000 & 0.000 \\
\hline
\end{tabular}

Compared with the control group, ${ }^{\Delta} \mathrm{P}<0.05$; compared with no cognitive dysfunction, ${ }^{\boldsymbol{\Lambda}} \mathrm{P}<0.05$. NSE, neuron-specific enolase; Bcl-2, B-cell lymphoma/leukemia-2.

Table 3 Correlation analysis of ATPase activity and serum NSE, S100B and Bcl-2 levels with seizure time, frequency and total MoCA score

\begin{tabular}{|c|c|c|c|c|c|c|}
\hline Indicators & \multicolumn{2}{|c|}{ Seizure time } & \multicolumn{2}{|c|}{ Seizure frequency } & \multicolumn{2}{|c|}{ MoCA score (total) } \\
\hline Hydrolysis rate of ATP & 0.668 & 0.000 & 0.569 & 0.001 & -0.602 & 0.001 \\
\hline Hydrolysis rate of ADP & 0.582 & 0.000 & 0.658 & 0.000 & -0.654 & 0.001 \\
\hline Hydrolysis rate of AMP & 0.586 & 0.003 & 0.710 & 0.000 & -0.404 & 0.007 \\
\hline NSE & 0.610 & 0.001 & 0.375 & 0.014 & -0.422 & 0.007 \\
\hline S100B & 0.428 & 0.007 & 4.474 & 0.005 & -0.612 & 0.000 \\
\hline $\mathrm{Bcl}-2$ & 0.475 & 0.006 & 6.010 & 0.000 & -0.547 & 0.003 \\
\hline
\end{tabular}

NSE, neuron-specific enolase; MoCA, Montreal Cognitive Assessment; ATP, adenosine triphosphate; ADP, adenosine diphosphate; AMP, adenosine monophosphate; PDE, phosphodiesterase; Bcl-2, B-cell lymphoma/leukemia-2.

\section{Comparison of serum NES, S100B and Bcl-2 levels between the two groups}

In the epilepsy group, the serum NES, S100B and Bcl-2 levels were significantly higher in patients with cognitive impairment than without $(\mathrm{P}<0.05)$. Also, as shown in Table 2, the serum NES, S100B and Bcl-2 levels of epileptic patients with and without cognitive impairment were significantly higher than those of the control group $(\mathrm{P}<0.05)$.

\section{Correlation analysis}

As shown in Table 3, Spearman's correlation analysis showed that the ATP, ADP, and AMP hydrolysis rates, PDE activity, and serum NES, S100B and Bcl-2 levels were positively correlated with seizure time and frequency $(\mathrm{P}<0.05)$, and negatively correlated with total MoCA score $(\mathrm{P}<0.05)$.

\section{Discussion}

In this study, 18 patients with epilepsy had cognitive dysfunction, with an incidence rate of $27.69 \%$. The seizure time and frequency of patients with cognitive impairment were significantly higher than those without cognitive impairment. MoCA total scores of patients with cognitive dysfunction were significantly lower than patients without cognitive dysfunction. The incidence of cognitive impairment among the epileptic patients in this study was slightly lower than the recently reported $30 \%$ to $40 \%$ in China (9), which may be related to regional differences and patient sample sizes. These results suggested that patients with epilepsy have significantly lower cognitive function than healthy individuals. Patients with epilepsy have a higher risk of cognitive dysfunction after seizures, and the risk may be higher in people with a longer time and high 
frequency of epileptic seizure. In addition, other studies (10) have confirmed that in addition to the time and frequency of seizures, electroencephalogram (EEG) performance can also indicate cognitive dysfunction. In this study, there were no EGG for some cases, so no further analysis was conducted on it. But we could also conclude that the severity index of epilepsy patients could reflect their cognitive function to a certain extent. At present, the specific mechanism of seizure-induced cognitive dysfunction has yet to be fully elucidated. Many scholars have analyzed the abnormal conditions of patients' brain regions from the perspective of MR imaging and have presented relevant explanations. The blood-oxygen-level-dependent (BOLD) signal is usually produced by spontaneous neural activity when the brain is in a resting state. Spontaneous abnormal discharge of neurons in the brains of epileptic patients can lead to the disorder of neurological function in the brain, and the BOLD signal deviates from the baseline more obviously than in healthy individuals, making it easy to detect the increase in the low-frequency amplitude value. An increase in the low-frequency amplitude of the brain region usually indicates that the activity in this region is facilitated, which is related to the seizure discharge stimulus. While a decrease in the low-frequency amplitude often indicates that normal brain function is inhibited, which is typical in patients with seizures and results in language disorders, memory loss, loss of consciousness, and other symptoms of cognitive impairment (11).

In this study, we measured the serum ATPase activity of the patients. The results showed that the ATP, ADP, and AMP hydrolysis rates and PDE activity of patients with cognitive impairment in the epilepsy group were significantly higher than those of patients without cognitive impairment. The serum indicators of above two were significantly higher than those in the control group, indicating that serum ATPase activity was increased in patients with seizures; it has been suggested that this increase might be related to cognitive dysfunction. Studies by Shan et al. (12) showed that the ATP, ADP, and AMP hydrolysis rates and PDE activity of elderly patients with epilepsy could show abnormal increases in the 5-10 minutes following a seizure, reaching a peak value at 20 minutes. Increased ATPase activity is believed to be involved in seizures. Hang et al. (13) clearly pointed out that the change of ATPase activity in patients with seizures was closely related to the degree of brain damage. They observed that abnormal synchronized excess firing by a large number of neurons during a seizure caused a large amount of biological energy to be consumed in the brain. Under this stress state, glycolytic metabolism, the energy supply pathway of the body is significantly enhanced, thus relieving the relative energy supply shortage caused by damage to the energy supply system of aerobic metabolism and providing energy for epileptic seizures (14). In the adenosine system, ATP is an energy product in the metabolic process, which can be released into ADP by the action of soluble PDE. Meanwhile, AMP is degraded by ADP through ADP hydrolase and hydrolyzed to adenosine by soluble 5 '-nucleotide enzyme. Thus, clinical scholars believe the adenosine system to be involved in the whole process of seizures. The longer the seizures, the higher the level of ATPase activity, which usually indicates that the disease is more severe (15). Moreover, the more severe the disease, the more obvious the cognitive impairment, which may explain the correlation between serum ATPase activity and cognitive function. Nevertheless, the specific reason still needs further exploration.

Besides, this study also measured the serum levels of NES, S100B and Bcl-2 in patients. The results showed that the serum levels of NES and Bcl-2 were significantly increased after seizures, which suggested that the increase might be related to cognitive dysfunction. NES typically exists specifically in neurons and neuroendocrine cells. When ischemia, hypoxia, poisoning, or injury occurs, NES can be released from cells in large quantities and enter the blood through the damaged blood-cerebrospinal fluid barrier. Therefore, NSE is considered to be highly sensitive for assessing the degree of brain tissue damage; As a specific brain protein, S100B mainly exists in neurons and glial cells of the central nervous system, which participates in some biological activities include neuronutrition, regulation of intracellular and extracellular calcium ion concentration, and participation in cell membrane differentiation and apoptosis (16). Niu et al. (17) found that serum S100B level was negatively correlated with scores of delayed memory and attention in patients with cognitive impairment caused by epilepsy. Hao et al. (18) showed that serum NES level in epileptic children with seizures lasting longer than 5 minutes was significantly higher than in children with seizures lasting less than 5 minutes. The increase in serum NES levels is believed to reflect the severity of seizures and disease. Meanwhile, Bcl-2 is an apoptotic gene that can promote neuronal cell apoptosis. A meta-analysis (19) pointed out that seizures can directly or indirectly result in morphological changes in the brain and death of neurons in susceptible areas, such as the hippocampus, which in turn 
promotes the increase of serum Bcl-2. Patients who suffered from various types of seizures and whose seizures last a longer time had higher serum bcl-2 levels. The analysis of Liu et al. (20) found that except for directional force and naming, serum Bcl-2 in epilepsy patients was correlated with word recall, delayed recall, and other MoCA-related factors. Recent research by Wang et al. (21) clearly showed that serum Bcl-2 in patients with epileptic seizures was significantly negatively correlated with the total MoCA score for cognitive function. The Spearman's correlation analysis in this study further confirmed that serum ATPase activity and levels of NES and Bcl-2 were related to seizure time and post-seizure cognitive function, which was consistent with the above views.

In conclusion, the cognitive function of patients with epilepsy is significantly lower than that of healthy individuals. Serum ATPase activity and NES, S100B and Bcl-2 levels are closely related to the seizure time and postseizure cognitive function. The above indicators should be closely monitored clinically, and timely measures to prevent and improve the cognitive function of the patient need to be taken. However, the sample size of this study was limited, and serum indexes were all measured by enzyme-linked immunosorbent method, which was not confirmed by other detection methods, because different methods may get different results. Besides, patients with other rare types of epilepsy were not analyzed. Therefore, relevant conclusions still need to be further confirmed by expanding the sample size and increasing dynamic monitoring results.

\section{Acknowledgments}

Funding: None.

\section{Footnote}

Reporting Checklist: The authors have completed the MDAR reporting checklist. Available at http://dx.doi.org/10.21037/ apm-20-1494

Data Sharing Statement: Available at http://dx.doi. org/10.21037/apm-20-1494

Conflicts of Interest: All authors have completed the ICMJE uniform disclosure form (available at http://dx.doi. org/10.21037/apm-20-1494). The authors have no conflicts of interest to declare.
Ethical Statement: The authors are accountable for all aspects of the work in ensuring that questions related to the accuracy or integrity of any part of the work are appropriately investigated and resolved. Written informed consent was obtained from each study participant before the initiation of the study procedures, and the study protocol and informed consent documents were reviewed and approved by the hospital Ethics Committees (No. 2018-053). All procedures performed in this study involving human participants were in accordance with the Declaration of Helsinki (as revised in 2013).

Open Access Statement: This is an Open Access article distributed in accordance with the Creative Commons Attribution-NonCommercial-NoDerivs 4.0 International License (CC BY-NC-ND 4.0), which permits the noncommercial replication and distribution of the article with the strict proviso that no changes or edits are made and the original work is properly cited (including links to both the formal publication through the relevant DOI and the license). See: https://creativecommons.org/licenses/by-nc-nd/4.0/.

\section{References}

1. Shu Y, Zhu C, Zeng M, et al. The protective effect of carbenoxolone on gap junction damage in the hippocampal CA1 area of a temporal lobe epilepsy rat model. Ann Transl Med 2019;7:624.

2. Braakman HM, van der Kruijs SJ, Vaessen MJ, et al. Microstructural and functional MRI studies of cognitive impairment in epilepsy. Epilepsia 2012;53:1690-9.

3. Guazzi M, Striano P. GABA strikes down again in epilepsy. Ann Transl Med 2019;7:57.

4. Lin XJ, Yang JB, Zhou Q, et al. Effect of valproate tablets on adenosine levels in elderly patients with epilepsy. Chinese Journal of Gerontology 2014;34:5349-350.

5. Wang LG, Zhai QX, Tang ZH, et al. Effect of elipcimide combined western drugs on serum level of neuron specific enolase in treating epilepsy children patients. Chinese Journal of Integrated Traditional and Western Medicine 2016;36:912-5.

6. Commission on classification and terminology of the international league against epilepsy. Proposal for revised classification of epilepsies and epileptic syndromes. Epilepsia 1989;30:389-99.

7. Gill DJ, Freshman A, Blender JA, et al. The Montreal cognitive assessment as a screening tool for cognitive 
impairment in Parkinson's disease. Mov Disord 2008;23:1043-6.

8. Wu YZ, Deng WJ, Xu GW, et al. Change of the contents of adenosine triphosphate in hippocampus as well as adenosine triphosphatase activity in serum in epileptic rats. Journal of Chinese Practical Diagnosis and Therapy 2017;31:632-5.

9. Ti Y, Zheng H. Epilepsy and cognitive disorders. Chinese General Practice 2017;20:1818-22.

10. Liao W, Zhang Z, Mantini D, et al. Dynamical intrinsic functional architecture of the brain during absence seizures. Brain Struct Funct 2014;219:2001-15.

11. Li SL, Qian RB, Fu XM, et al. Alteration of cerebral small world network in refractory epilepsy patients: a restingstate functional magnetic resonance imaging. Chinese Journal of Neurology 2015;48:677-80.

12. Shan Q, Wang SX, Li PD, et al. Dynamic expression of adenosine and adenylase in elderly patients with epilepsy. Chinese Journal of Gerontology 2013;33:5517-9.

13. Hang Y, Zhang H, Yun YL, et al. Relation between plasma acidic phospholipid and brain damage after epileptic attack. Chinese Journal of Geriatric Heart Brain and Vessel Diseases 2017;19:192-4.

14. Liu ZY, Wang LK, Wu GF. Expressions of cAMP response element binding protein and phosphorylated cAMP response element binding protein in the hippocampus of drug resistant temporal lobe epileptic rats. Chinese Journal of Neurology 2016;49:630-4.

15. Wang X, Zhang Y, Wei G, et al. Mechanism of Nucleoside
Triphosphate Diphosphohydrolase-1-Associated Imbalance in Adenosine Diphosphate Degradation, B-Cell Activation, and Related Injury During Acute AntibodyMediated Rejection. Transplant Proc 2018;50:1292-7.

16. Ji X, Hua Y, Zhang XJ. Clinical significance of combined detection of IGF-1 and NSE in serum of children with epilepsy. The Journal of Practical Medicine 2016;32:650-2.

17. Niu Q, Shen QY, Wang SY, et al. The correlation between serum S100B protein level and cognitive function in epilepsy patients. Journal of Apoplexy and Nervous Diseases 2019;36:60-3.

18. Hao WH, Xu BY, Wang LH. The correlation of serum NSE and BDNF with epileptic seizures and development infants. Journal of Chengdu Medical College 2016;11:466-8.

19. Kikuyama H, Hanaoka T, Kanazawa T, et al. The mechanism of anti-epileptogenesis by levetiracetam treatment is similar to the spontaneous recovery of idiopathic generalized epilepsy during adolescence. Psychiatry Investig 2017;14:844.

20. Liu YH, Chang ZD, Niu B. Characteristics of cognitive impairment in patients with epilepsy and the relationship between serum Bcl-2 levels and cognitive function. Guangdong Medical Journal 2016;37:94-8.

21. Wang Y, Feng JG. Correlation between cognitive function and serum Bcl-2 and IGF-1 in elderly patients with epilepsy. Chinese Journal of Gerontology 2018;38:1151-3.

(English Language Editor: J. Reynolds)
Cite this article as: Zhang Y, Zhang S, Ji Y, Yang X, Liu P, Yu G. Relationship of serum ATPase activity and the levels of neuronspecific enolase, S100B and B-cell lymphoma/leukemia-2 with cognitive function after epileptic seizure. Ann Palliat Med 2020;9(5):3366-3372. doi: 10.21037/apm-20-1494 